\begin{tabular}{l|l} 
2. To: (Receivingorganization) & 3. From: corigi \\
300 Area Liquid Effluents & Process Syst \\
ProcesS Engineering & \\
\hline $\begin{array}{l}\text { 5. Proj./Prog./Dept./Div.: } \\
\text { L-045H }\end{array}$ & $\begin{array}{l}\text { 6. Cog. Engr.: } \\
\text { RJ MCCARTHY }\end{array}$ \\
\hline
\end{tabular}

8. Originator Renarks:

Key Words: Treated Effluent Disposal Facility, TEDF,

4. Related EDT No.:

NA

7. Purchase Order No.:

NA

9. Equip./Component Mo.:

NA

10. System/Bldg./Facility:

300 Area/TEDF

11. Receiver Remarks:

12. Mojor Mesm. Owg. Mo.:

NA

13. Permit/Permit Application No.: NA

14. Required Response Date:

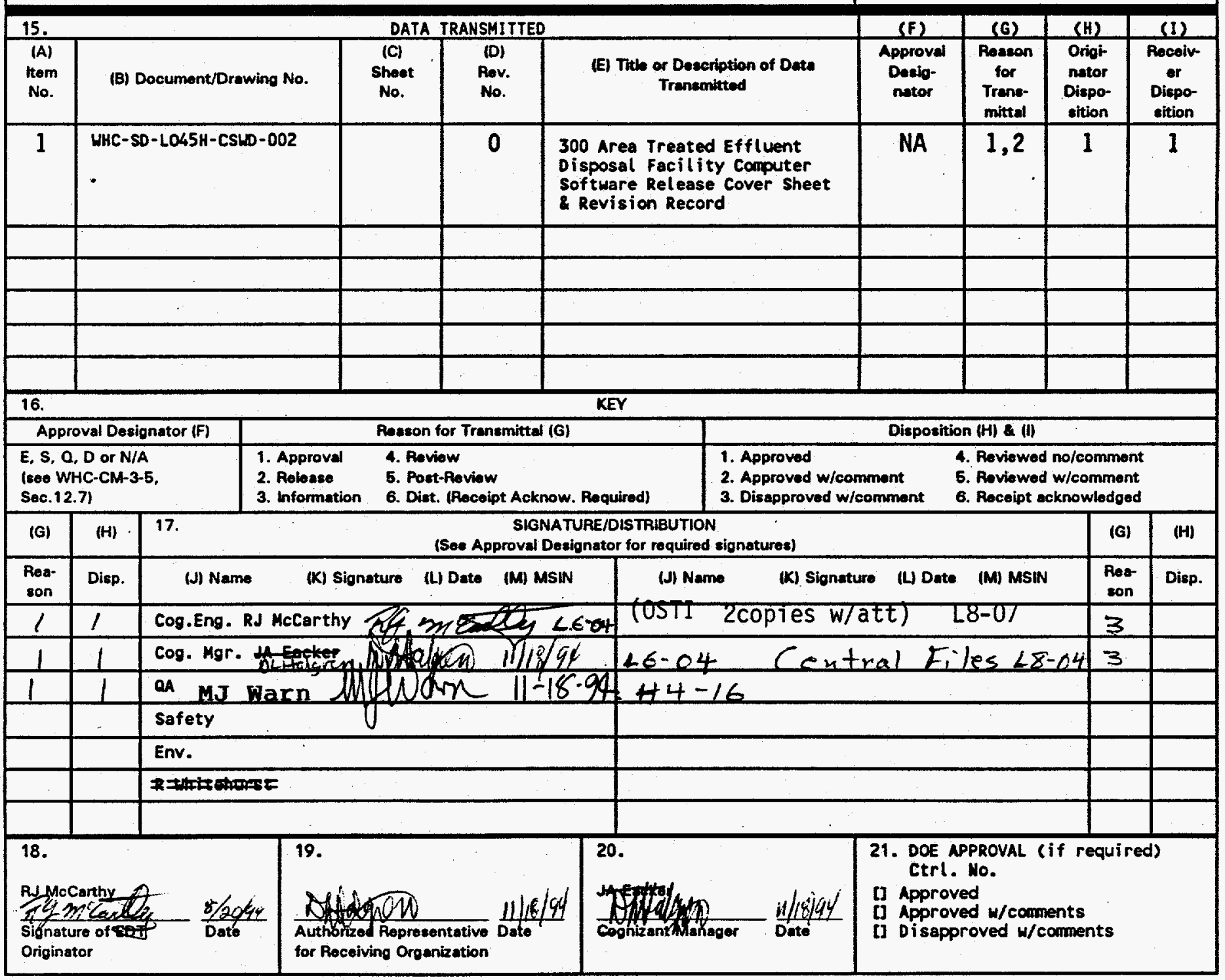

BD-7400-172-2 (04/94) GEF097 


\section{DISCLAIMER}

Portions of this document may be illegible in electronic image products. Images are produced from the best available original document. 


\section{RELEASE AUTHORIZATION}

Document Number: WHC-SD-LO45H-CSWD-002, Revision 0

Document Title: $\quad 300$ Area Treated Effluent Disposal Facility Computer Software Release Cover Sheet and Revision Record

Release Date: $\quad$ November 18, 1994

This document was reviewed following the procedures described in WHC-CM-3-4 and is:

APPROVED FOR PUBLIC RELEASE

WHC Information Release Administration Specialist:

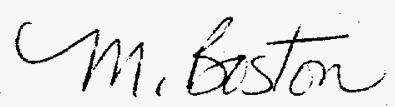

\section{M.N. Boston}

TRADEMARK DISCLAIMER. Reference herein to any specific commercial product, process, or service by trade name, trademark, manufacturer, or otherwise, does not necessarily constitute or imply its endorsement, recommendation, or favoring by the United States Government or any agency thereof or its contractors or subcontractors.

This report has been reproduced from the best available copy. Available in paper copy and microfiche. Printed in the United States of America. Available to the U.S. Department of Energy and its contractors from:

U.S. Department of Energy

office of Scientific and Technical Information (OSTI)

P.0. Box 62

Oak Ridge, TH 37831

Telephone: (615) 576-8401

Available to the public from:

U.S. Department of Comerce National Technical Information Service (NT1S)

5285 Port Royal Rosd

Springfield, VA 22161

Telephone: (703) $487-4650$ 


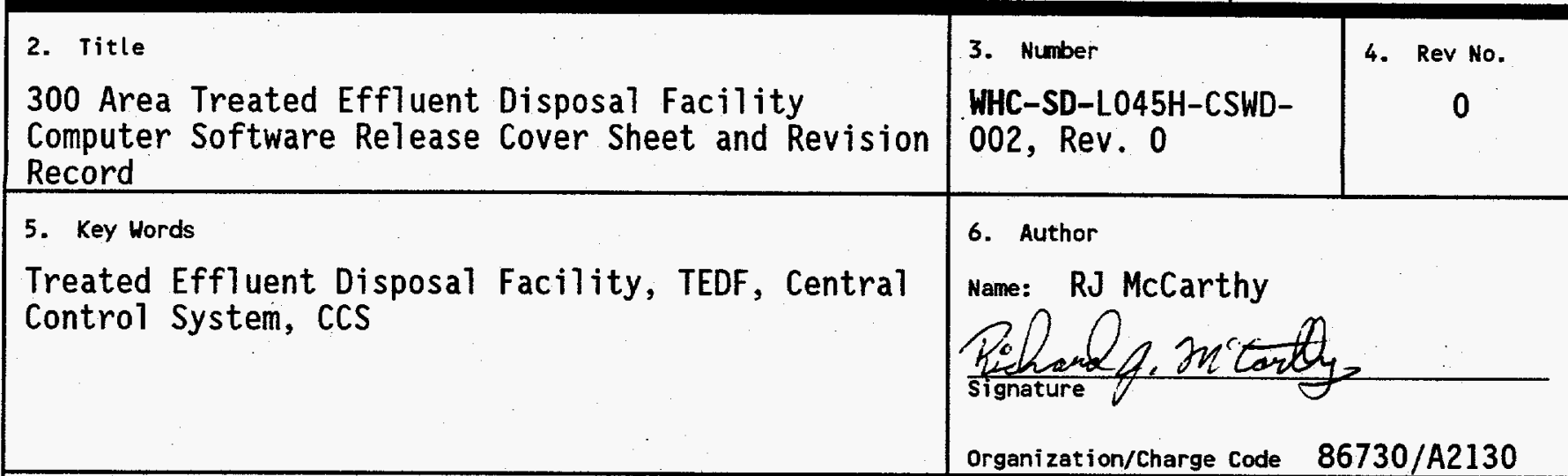

7. Abstract

This Supporting Document contains the Computer Software Release Cover Sheet and Revision Records for the 300 Area Treated Effluent Disposal Facility (TEDF).

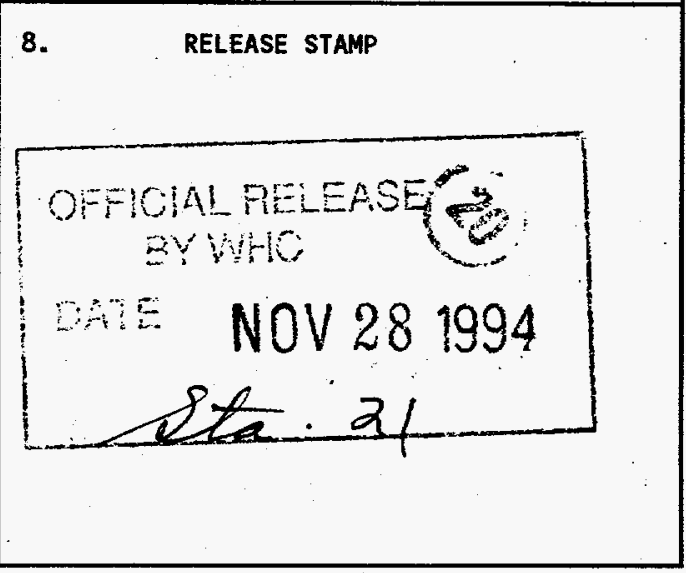




\title{
300 Area Treated Effluent Disposal Facility
}

\section{Computer Software Release Cover Sheet \&}

\author{
Revision Record
}

\section{DISCLAIMER}

This report was prepared as an account of work sponsored by an agency of the United States Government. Neither the United States Government nor any agency thereof, nor any of their employees, makes any warranty, express or implied, or assumes any legal liability or responsibility for the accuracy, completeness, or usefulness of any information, apparatus, product, or process disclosed, or represents that its use would not infringe privately owned rights. Reference herein to any specific commercial product, process, or service by trade name, trademark, manufacturer, or otherwise does not necessarily constitute or imply its endorsement, recommendation, or favoring by the United States Government or any agency thereof. The views and opinions of authors expressed herein do not necessarily state or reflect those of the United States Government or any agency thereof. 


\section{RELEASE COVER SHEET \& REVISION RECORD}

1. Software ID (Name):

Revision:

1

2. Release Type: [X] Initial Release [ ]

Approval Level Change: E S Q D Sfty Class $Q$

3. Abstract

Initial WHC release of the 300 Area TEDF software. The previous REV 0 was controlled by $\mathrm{CH} 2 \mathrm{M} \mathrm{Hill}$ who developed the software.

4. Software files (or attach directory listing)

See Attached Listing

5. Software files record storage media and location

Backup 1: $88 \mathrm{Meg}$. Syquest Cartridge located in Mo744

Backup 2: $88 \mathrm{Meg}$. Syquest Cartridge located in Mo744

\begin{tabular}{|c|c|c|c|c|}
\hline $\begin{array}{l}6 . \\
\text { Documentation }\end{array}$ & Title & \multicolumn{2}{|c|}{ Document Number } & Rev \\
\hline Requirements & $\begin{array}{l}\text { L-045H Project TEDF } \\
\text { Computer Software } \\
\text { Requirements } \\
\text { Specification }\end{array}$ & \multicolumn{2}{|c|}{$\begin{array}{l}\text { WHC-SD-L045H- } \\
\text { CSRS-001 }\end{array}$} & 1.0 \\
\hline Design & $\begin{array}{l}\text { L-045H Project TEDF } \\
\text { Computer Software Design } \\
\text { Description }\end{array}$ & \multicolumn{2}{|l|}{$\begin{array}{l}\text { WHC-SD-ETF- } \\
\text { CSDD-001 }\end{array}$} & 0.0 \\
\hline Validation & $\begin{array}{l}\text { Acceptance Test Procedure } \\
\text { For The } 300 \text { Area Treated } \\
\text { Effluent Disposal } \\
\text { Facility Process Control } \\
\text { System }\end{array}$ & \multicolumn{2}{|c|}{$\begin{array}{l}\text { WHC-SD-L045H- } \\
\text { ATP-002 }\end{array}$} & 0.0 \\
\hline User & $\begin{array}{l}\text { L-045H Project TEDF } \\
\text { Computer Software User } \\
\text { Document }\end{array}$ & \multicolumn{2}{|l|}{$\begin{array}{l}\text { WHC-SD-ETF- } \\
\text { CSUD-001 }\end{array}$} & 0.0 \\
\hline $\begin{array}{r}\text { Configuration } \\
\text { Control }\end{array}$ & $\begin{array}{l}\text { L-045H Project TEDF } \\
\text { Computer Software } \\
\text { Configuration Management } \\
\text { Plan }\end{array}$ & \multicolumn{2}{|l|}{$\begin{array}{l}\text { WHC-SD-ETF- } \\
\text { CSCM-001 }\end{array}$} & 0.0 \\
\hline 7. Environment & Description & Rev & \multicolumn{2}{|c|}{$\begin{array}{l}\text { Approval Lvl/Sfty } \\
\text { Class }\end{array}$} \\
\hline Hardware & $\begin{array}{l}\text { Two PC based DOS Computers } \\
\text { Two Programmable Logic } \\
\text { Controllers (Siemens } \\
545 \text { ) }\end{array}$ & $\begin{array}{l}N / A \\
2.1\end{array}$ & 4 & \\
\hline
\end{tabular}




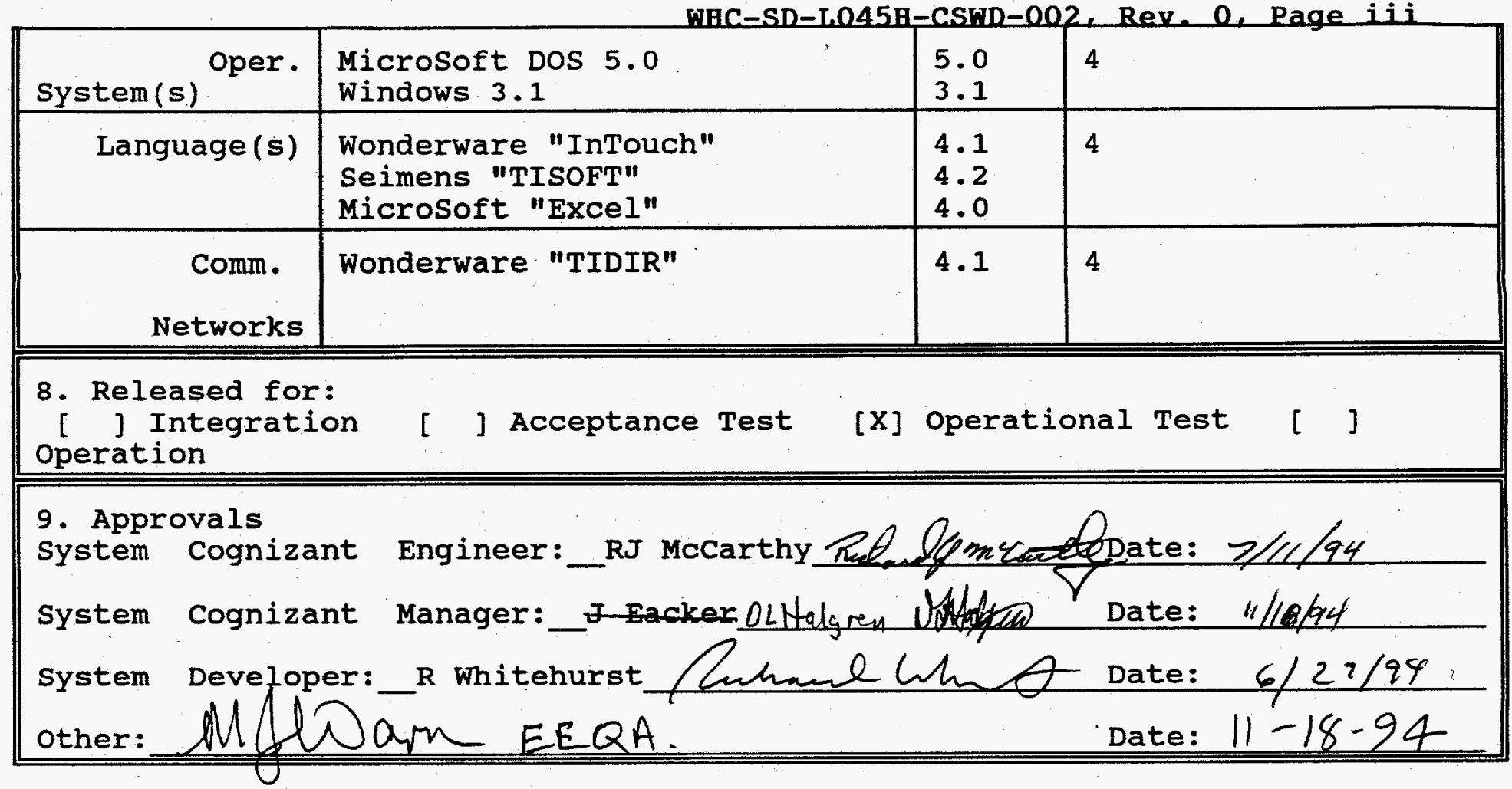




\section{RELEASE COVER SHEET AND REVISION 300 AREA TEDF FILE LIST}

Volume in drive $C$

Directory of C: ITEDF

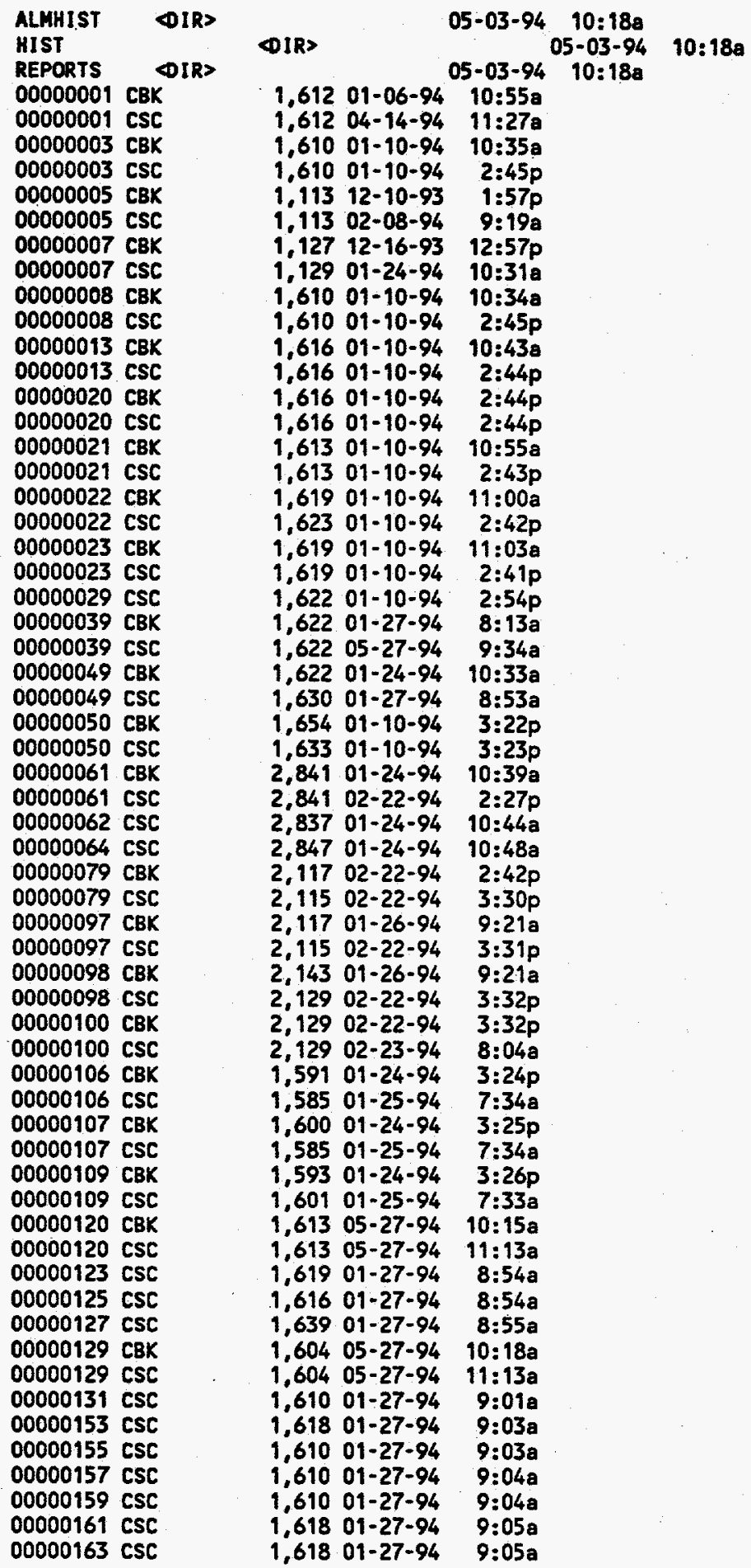




\section{RELEASE COVER SHEET AND REVISION 300 AREA TEDF FILE LIST}

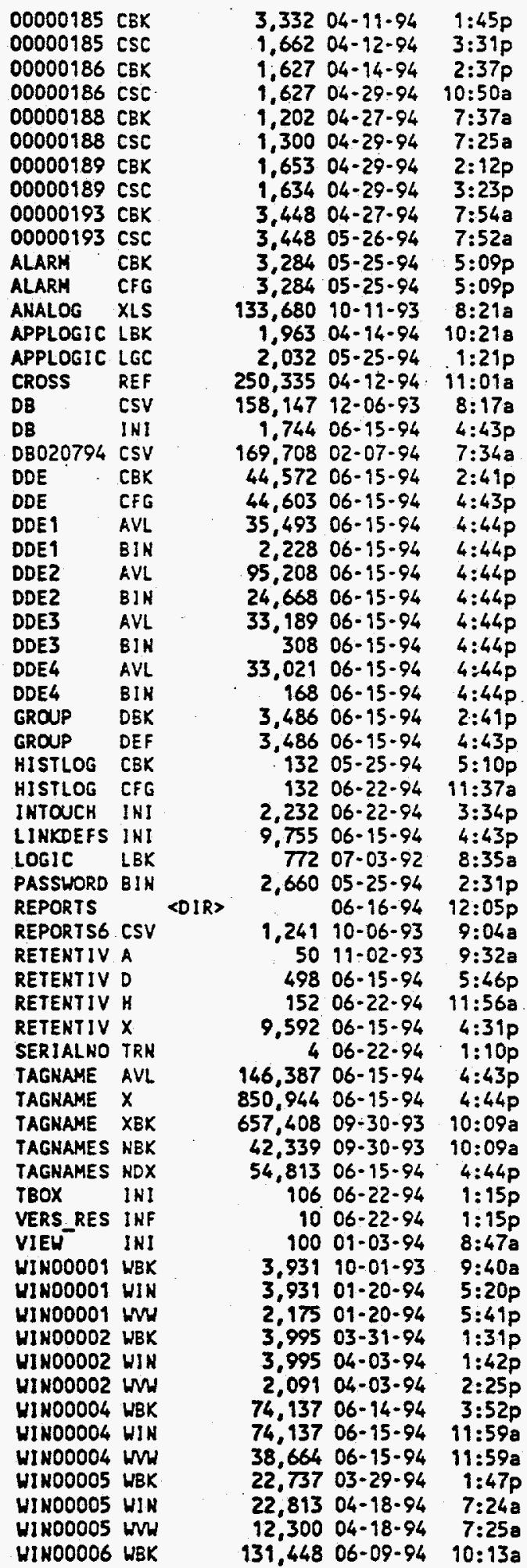




\section{RELEASE COVER SHEET AND REVISION 300 AREA TEDF FILE LIST}

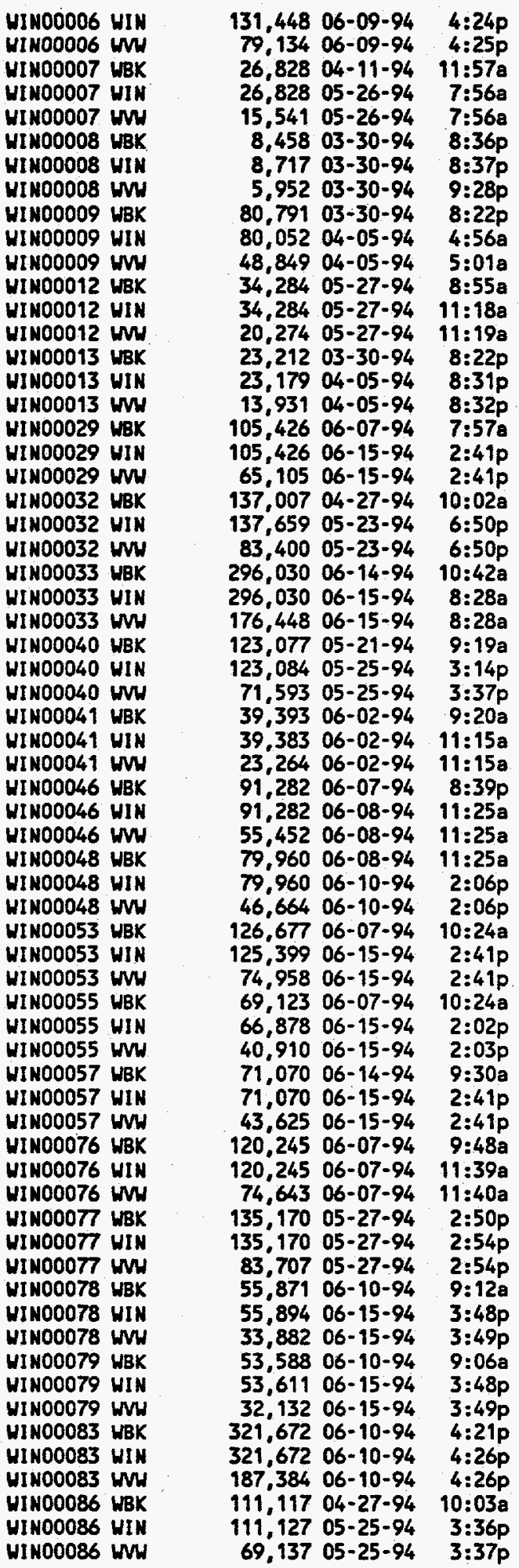




\section{RELEASE COVER SHEET AND REVISION 300 AREA TEDF FILE LIST}

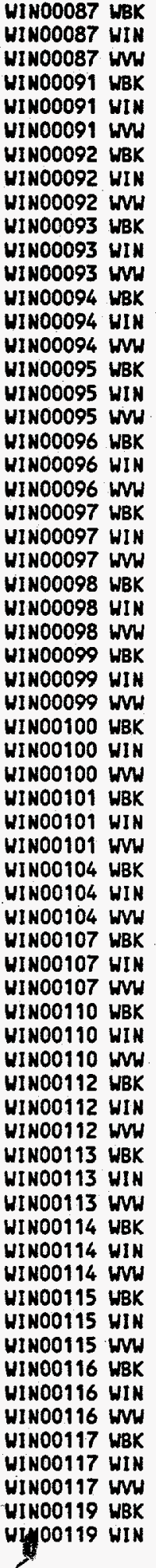

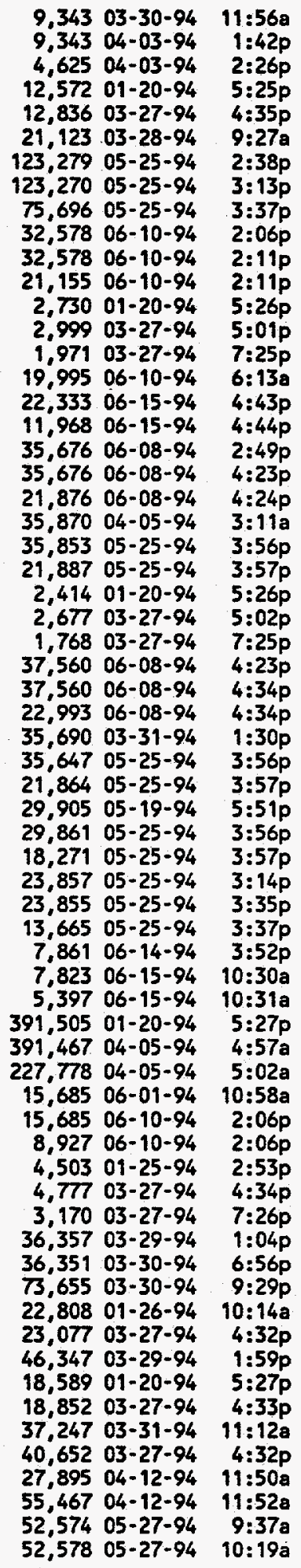




\section{RELEASE COVER SHEET AND REVISION 300 AREA TEDF FILE LIST}

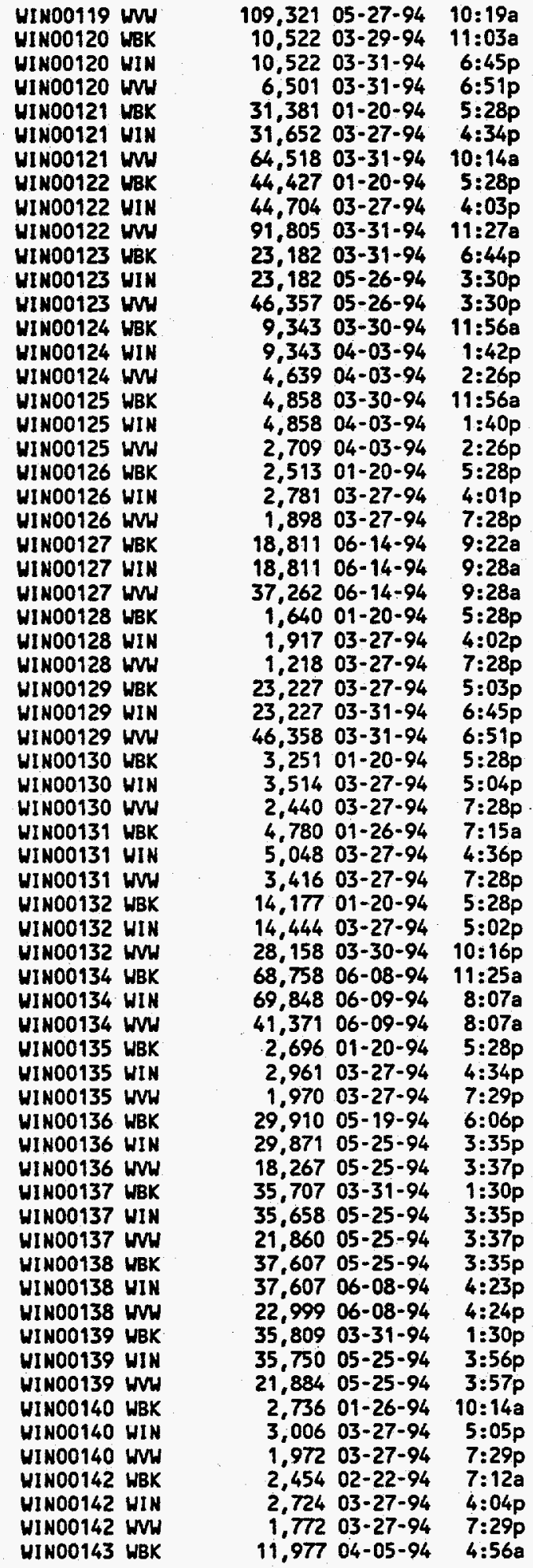




\section{RELEASE COVER SHEET AND REVISION 300 AREA TEDF FILE LIST}

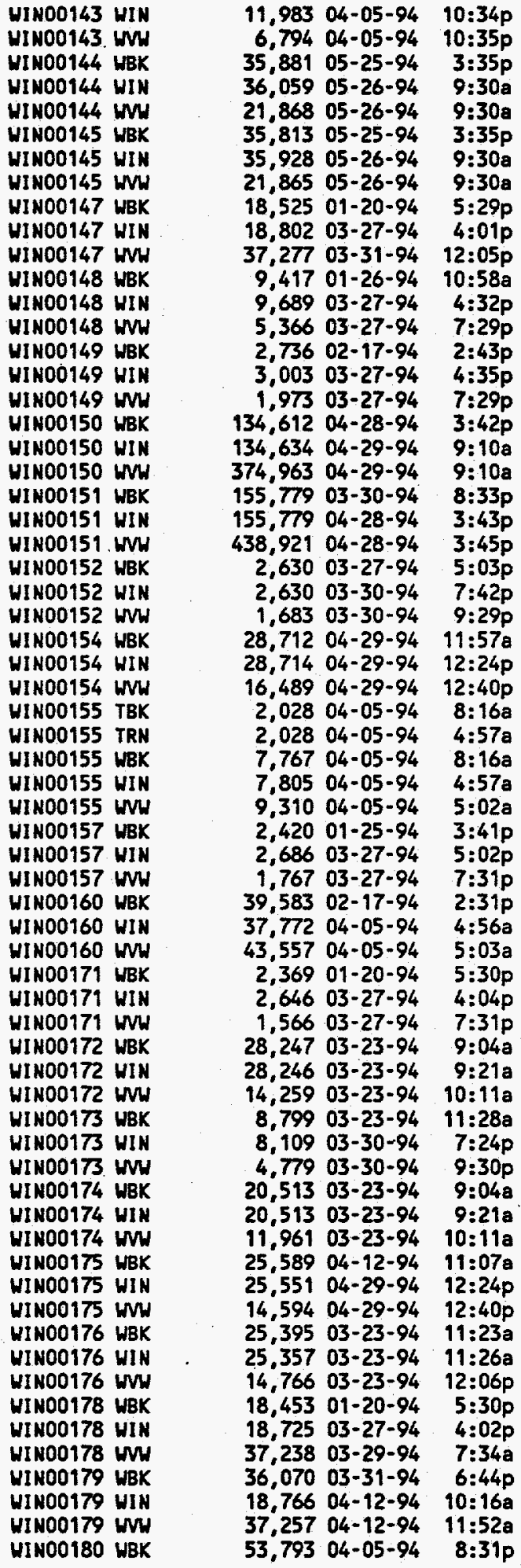




\section{RELEASE COVER SHEET AND REVISION 300 AREA TEDF FILE IIST}

\begin{tabular}{|c|c|c|}
\hline $\begin{array}{l}\text { WIN00180 } \\
\text { WINO0180 } \\
\text { WIN00181 } \\
\text { WIN00181 } \\
\text { WINO0181 } \\
\text { WH } \\
\text { WHWWS } \\
\text { WW WDWS }\end{array}$ & $\begin{array}{l}\text { WIN } \\
\text { WNH } \\
\text { WBK } \\
\text { WIN } \\
\text { WWH } \\
\text { INI } \\
\text { NBK } \\
\text { NDX }\end{array}$ & 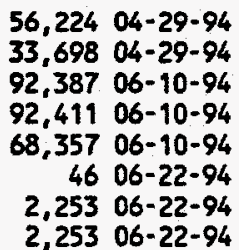 \\
\hline
\end{tabular}

Directory of C:ITEDFIREPORTS

$\begin{array}{llrll}\text { REPORT } & \text { XLM } & 4,691 & 04-28-94 & 3: 26 p \\ \text { REPORT } & \text { XLS } & 12,499 & 04-28-94 & 3: 21 p \\ \text { REPORT } & \text { XLT } & 16,690 & 04-28-94 & 1: 41 p \\ \text { STARTMAC XLM } & 2,223 & 04-27-94 & 2: 36 p\end{array}$

